Claremont Colleges

Scholarship@ Claremont

All HMC Faculty Publications and Research

HMC Faculty Scholarship

5-1-1995

\title{
Distortion and Evolution of a Localized Vortex in an Irrotational Flow
}

Joseph F. Lingevitch

Northwestern University

Andrew J. Bernoff

Harvey Mudd College

\section{Recommended Citation}

Distortion and evolution of a localized vortex in an irrotational flow. Joseph F. Lingevitch and Andrew J. Bernoff, Phys. Fluids 7, 1015 (1995).

This Article is brought to you for free and open access by the HMC Faculty Scholarship at Scholarship @ Claremont. It has been accepted for inclusion in All HMC Faculty Publications and Research by an authorized administrator of Scholarship @ Claremont. For more information, please contact scholarship@cuc.claremont.edu. 


\title{
Distortion and evolution of a localized vortex in an irrotational flow
}

\author{
Joseph F. Lingevitch and Andrew J. Bernoff \\ Department of Engineering Sciences and Applied Mathematics, Northwestern University, Evanston, \\ Illinois 60208
}

(Received 18 October 1994; accepted 27 January 1995)

\begin{abstract}
This paper examines the interaction of an axisymmetric vortex monopole, such as a Lamb vortex, with a background irrotational flow. At leading order, the monopole is advected with the background flow velocity at the center of vorticity. However, inhomogeneities of the flow will cause the monopole to distort. It is shown that a shear-diffusion mechanism, familiar from the study of mixing of passive scalars, plays an important role in the evolution of the vorticity distribution. Through this mechanism, nonaxisymmetric vorticity perturbations which do not shift the center of vorticity are homogenized along streamlines on a $\mathrm{Re}^{1 / 3}$ time scale, much faster than the Re decay time scale of an axisymmetric monopole. This separation of time scales leads to the quasisteady evolution of a monopole in a slowly varying flow. The asymptotic theory is verified by numerically computing the linear response of a Lamb monopole to a time-periodic straining flow and it is shown that a large amplitude, $Q\left(\operatorname{Re}^{1 / 3}\right)$, distortion results when the monopole is forced at its resonant frequency. (c) 1995 American Institute of Physics.
\end{abstract}

\section{INTRODUCTION}

Localized vortices are commonly observed in large Reynolds number fluid flows and in many instances appear to be robust structures which persist for long times compared to the vortex turnover time. Geophysical flows present numerous examples of large Reynolds number vortices including vortex monopoles and dipoles in planetary oceans and atmospheres..$^{1-3}$ Numerical simulations of turbulent flows show the emergence of localized regions of vorticity. ${ }^{4-7}$ Vortices are also observed in moderate Reynolds number laboratory experiments such as impulsively started jets and flows past bluff bodies ${ }^{2,3,8-10}$ (e.g., flow past a cylinder).

Two-dimensional (2-D) models have been useful in understanding flows with vorticity. The frequent observation of vortices in fluids suggests the existence of stable vortex solutions to the 2-D vorticity equations. Indeed, it has been proven by Dritschel that axisymmetric, inviscid monopoles with monotonic vorticity distributions are Lyapunov stable. ${ }^{11}$ Another characteristic observed in these flows is that the vortices tend to be nearly circular. This observation has been supported by the inviscid numerical simulations of Melander et al. ${ }^{12}$ who have argued that isolated, smooth distributions of vorticity will axisymmetrize. We have previously demonstrated that a large Reynolds number Lamb monopole is linearly stable, relaxing to an axisymmetric state on a $\mathscr{C}\left(\operatorname{Re}^{1 / 3}\right)$ shear-diffusion time scale. ${ }^{13}$ The presence of an external flow, however, may perturb the streamlines of a vortex, leading to a nonaxisymmetric distortion.

Several inviscid models for the distortion of a monopole in a straining flow exist; Moore and Saffman ${ }^{14}$ showed elliptical vortex patch solutions existed in the presence of sufficiently weak external strain. Kida ${ }^{15}$ generalized these solutions and showed they may rotate, nutate or extend indefinitely for sufficiently strong external flows. Dritschel ${ }^{16}$ studied the stability of these solutions; he noted that resonance between the rotation rate of the ellipse and the oscillation frequency of a boundary perturbation can lead to instability. Legras and Dritschel ${ }^{17,18}$ study the inviscid evolution of monopoles with monotonic profiles in an external straining flow. For small straining amplitudes, a monopole oscillates between axisymmetric and elongated shapes. Larger amplitudes lead to the stripping of vorticity from the outer edge of the monopole.

In large Reynolds number simulations, McWilliams ${ }^{6}$ has observed the emergence and interaction of vortex monopoles and dipoles from decaying turbulence. The interactions range from weak nonaxisymmetric distortions to strong interactions such as vortex merging and stripping. In this paper, we consider the large Reynolds number dynamics of an axisymmetric monopole in a perturbing irrotational flow such as that which might be induced by distant vortices or boundary conditions. Our goal is to obtain a fundamental description of this interaction.

Previously, the viscous regularization of an initial point vortex in a nonuniform irrotational flow has been studied by Ting and his collaborators ${ }^{19,20}$ and extended by Gunzburger. ${ }^{21}$ They showed that, to leading order, a monopole is advected at the velocity of the irrotational background flow evaluated at the center of the vortex. They found an oscillatory correction to this motion, which we will argue can be eliminated by a coordinate change. They also showed that a weak external straining flow induces a quadrupolar (i.e., slightly elliptical) distortion of the axisymmetric vortex.

We extend the work of Ting and his collaborators in a new formalism that adds to their description of a decaying vortex in two respects. First, we utilize a coordinate system attached to the vorticity centroid. The vortex velocity is determined by momentum conservation (see Saffman ${ }^{9}$ ) and to leading order is advected at the irrotational flow velocity evaluated at the centroid, in agreement with the "coarse grained" result of Ting and Tung. ${ }^{19}$ This method yields the form of the velocity corrections as well, allowing an estimation of their magnitude. Second, we incorporate into the perturbation theory the dissipative effects of a shear-diffusion mechanism that was overlooked in the analysis of Ting and Tung. Through shear and viscous diffusion, vorticity pertur- 
bations are homogenized along the streamlines of the monopole on a $\operatorname{Re}^{1 / 3}$ time scale. This yields a description of a decaying vortex on three distinct time scales; the $\mathbb{C}(1)$ vortex turnover time, the $\mathcal{Q}\left(\mathrm{Re}^{1 / 3}\right)$ shear-diffusion time scale, and the $Q(\operatorname{Re})$ viscous decay time scale.

In the next section, we formulate the problem of a localized vortex in an irrotational flow and calculate the translational velocity of a monopole. The vorticity equation is then transformed into a reference frame that propagates with the monopole velocity yielding an inhomogeneous equation for the distortion of the monopole. In Sec. III, the sheardiffusion mechanism is reviewed and the distortion of the monopole is computed in the limit of large Reynolds number. In Sec. IV, the asymptotic theory is validated by a numerical solution of the linearized vorticity equation for a Lamb monopole in a time-periodic straining flow. The frequency response of the monopole is computed and compared with the related problem of the mixing of a passive scalar. In Sec. $\mathrm{V}$, we compare our results in detail with the previous research of Ting et al. and Gunzburger and qualitatively with the inviscid models of a vortex in a straining flow. Section VI summarizes our results and suggests some avenues for further research.

\section{FORMULATION OF PROBLEM}

In this section, the 2-D Navier-Stokes equations are formulated to study a localized monopole in an external irrotational flow. The velocity of a monopole is calculated utilizing the conservation of momentum and the vorticity equation is transformed into a reference frame that moves with the monopole velocity.

\section{A. Governing equations}

The vorticity formulation of the Navier-Stokes equations is particularly suited to describing a localized vortex monopole. ${ }^{8,9}$ In $2 \mathrm{D}$, the vorticity is a scalar quantity proportional to the local angular velocity of the fluid and is equal to the curl of the velocity field, $\omega=(\nabla \times \mathbf{u}) \cdot \hat{k}$, where $\hat{k}$ is a unit vector perpendicular to the plane of the fluid. The vorticity obeys an advection-diffusion equation,

$$
\omega_{t}+\mathbf{u} \cdot \nabla \omega=\frac{1}{\operatorname{Re}} \nabla^{2} \omega,
$$

where $\operatorname{Re}=D^{2} /(\bar{T} \nu)$ is the nondimensional Reynolds number. Lengths are nondimensionalized on the vortex diameter, $D$; time is scaled by the vortex turnover time, $\bar{T}$; and $\nu$ is the kinematic viscosity of the fluid. Incompressibility is satisfied by introducing a streamfunction, $\mathbf{u}(\mathbf{x}, t)=\nabla \psi(\mathbf{x}, t) \times \hat{k}$. The streamfunction is related to the vorticity by a Poisson equation,

$$
\nabla^{2} \psi=-\omega
$$

The irrotational component of the streamfunction is determined by specifying boundary conditions on Eq. (2).

\section{B. Axisymmetric vortices}

Axisymmetric distributions of vorticity will form the basis of our perturbation expansion in Sec. III. Such monopoles

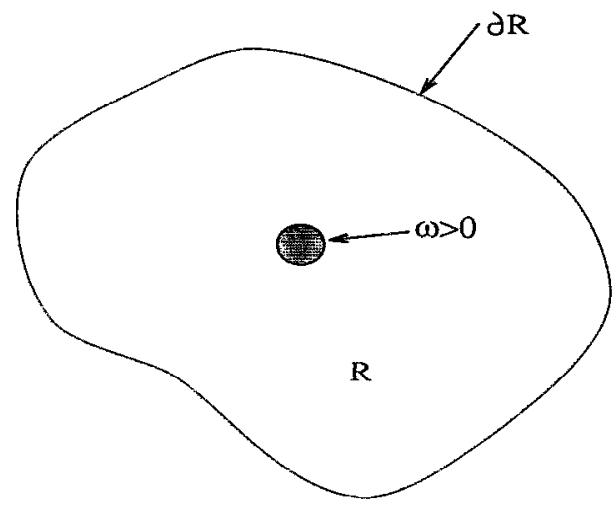

FIG. 1. Schematic illustration showing a localized vortex monopole in an external irrotational flow. The flow in the vicinity of the monopole is a supcrposition of the flow induced by the monopole itself plus an irrotational component induced by other vorticity distributions external to the domain $\mathbf{R}$ or boundary conditions on the fluid.

are exact solutions of Eq. (1). They possess circular streamlines along which the vorticity is constant and decay on the Reynolds number time scale due to the radial diffusion of vorticity. The Lamb vortex is a particular example with exponentially localized vorticity and corresponding streamfunction,

$$
\begin{aligned}
& \omega(\mathbf{x}, t) \equiv W(r, T)=\frac{1}{4 \pi T} \exp \left(\frac{-r^{2}}{4 T}\right), \\
& \psi(\mathbf{x}, t) \equiv \Psi(r, T)=-\frac{1}{2 \pi} \int_{0}^{r}\left[1-\exp \left(\frac{-r^{2}}{4 T}\right)\right] \frac{d r}{r},
\end{aligned}
$$

where $T$ is time scaled by the Reynolds number (i.e., $T=t$ / $\mathrm{Re})$. It is the viscous regularization of an isolated point vortex of unit strength. Since axisymmetric localized monopoles decay toward Gaussian profiles, ${ }^{20}$ it is natural to consider the Lamb vortcx as our basic monopolc solution. In the following, we compute the velocity and distortion of such a monopole in the presence of an external flow.

\section{Vortex velocity}

The first moment of a vorticity distribution is analogous to the linear momentum of the vortex. ${ }^{9}$ The conservation of this moment allows the velocity of the vortex centroid to be determined as an integral of the background irrotational flow weighted by the vorticity of the monopole. Below we derive an expression for the velocity of the monopole centroid.

Consider a simply connected region of fluid, $\mathscr{R}$, containing a single exponentially localized monopole (see Fig. 1). We assume the vorticity is exponentially decaying on a scale comparable to the characteristic radius of the vortex, which is small compared to the size of the domain $\mathscr{B}$. The centroid is defined by

$$
\mathbf{X}(t)=\frac{1}{\Gamma} \int_{\mathscr{B}} \mathbf{x} \omega d \mathbf{x}
$$

where $\Gamma=\int_{\mathscr{B}} \omega d \mathbf{x}$ is the integrated vorticity (a constant) of the monopole. The velocity of the centroid then follows ${ }^{9}$ from multiplying (1) by $\mathbf{x}$ and integrating over the region 3 , 


$$
\frac{d \mathbf{X}}{d t}=\frac{1}{\Gamma} \int_{S z} \mathbf{u} \omega d \mathbf{x}
$$

Utilizing the linear relationship between the vorticity and streamfunction, we decompose the fluid velocity (streamfunction) into a background component, $\mathbf{u}^{B}\left(\psi^{B}\right)$ and a component induced by the vorticity within $\mathscr{R}, \mathbf{u}^{\omega}\left(\psi^{\omega}\right)$,

$$
\psi(\mathbf{x}, t)=\psi^{B}(\mathbf{x}, t)+\psi^{\omega}(\mathbf{x}, t) .
$$

The vorticity induced component, $\psi^{\omega}$, is given by a convolution integral of the vorticity with the free space Green's function of the Poisson equation,

$$
\psi^{\omega}(\mathbf{x}, t)=-\frac{1}{4 \pi} \int_{9 g} \log \left|\mathbf{x}-\mathbf{x}^{\prime}\right|^{2} \omega\left(\mathbf{x}^{\prime}, t\right) d \mathbf{x}^{\prime} .
$$

The background component, $\psi^{\beta}$, is an irrotational flow, uniquely specified in $\mathscr{B}$ by its value on the boundary. From this decomposition, we split the integral in Eq. (5) into two pieces,

$$
\frac{d \mathbf{X}}{d t}=\frac{1}{\Gamma}\left(\int_{\mathscr{B}} \mathbf{u}^{B} \omega d \mathbf{x}+\int_{\mathscr{B}} \mathbf{u}^{\omega} \omega d \mathbf{x}\right) .
$$

The second integral in (8) vanishes; to show this we rewrite $\omega$ in the second integral as the $z$ component of the curl of $\mathbf{u}^{\omega}$. Utilizing the divergence theorem, the second integral becomes

$$
\int_{\mathscr{B}} u_{j}^{\omega} \omega d \mathbf{x}=\oint_{\partial \mathscr{B}} \hat{n}_{i} M_{i j} d s
$$

where the right-hand side of (9) is a counterclockwise line integral around $\partial \mathscr{F}, d s$ is an arclength, and $\hat{n}$ is an outward pointing normal to the contour. The subscript indices on the vectors denote the Cartesian components and the matrix $M_{i j}$ is given by

$$
\mathbf{M}=\left[\begin{array}{cc}
u v & -\left(u^{2}-v^{2}\right) / 2 \\
-\left(u^{2}-v^{2}\right) / 2 & -u v
\end{array}\right],
$$

where $\mathbf{u}^{\omega}=u \hat{\imath}+v \hat{\mathrm{j}}$. Due to the exponential localization of the vorticity, the integration contour in Eq. (9) can be extended to a contour at infinity with exponentially small error. Using the far-field behavior of the velocity, $\left|\mathbf{u}^{\omega}\right| \approx \Gamma / r$, shows that the line integral vanishes yielding

$$
\mathbf{V} \equiv \frac{d \mathbf{X}}{d t}=\frac{1}{\Gamma} \int_{B B} \mathbf{u}^{B} \omega d \mathbf{x}
$$

We now evaluate Eq. (11) to obtain a series expansion for the velocity of a monopole. In terms of a polar coordinate system centered at the vorticity centroid, $\mathbf{x}^{\prime}=\mathbf{x}-\mathbf{X}(t)$, the streamfunction of the external flow, which is harmonic, has a Taylor expansion about the vorticity centroid,

$\psi^{B}\left(\mathbf{x}^{\prime}+\mathbf{X}(t), t\right)=\psi^{B}(\mathbf{X}(t))+\left(\frac{1}{\epsilon} \sum_{n=1}^{\infty}(\epsilon r)^{n} a_{n} e^{i n \theta}+\right.$ c.c. $)$

where $r$ is the magnitude of the vector $\mathbf{x}^{\prime}$ (i.e., $r=\left|\mathbf{x}^{\prime}\right|$ ) and the Taylor expansion coefficients, $a_{n}(\mathbf{X}(t), t)$, depend on the location of the vortex and time. A scaling parameter, $\epsilon$, has been introduced into the streamfunction to characterize velocity fields whose length scale is large compared to the characteristic size of the vortex. From Eq. (12), the background velocity in Cartesian coordinates is

$$
\begin{aligned}
\mathbf{u}^{B}\left(\mathbf{x}^{\prime}+\mathbf{X}(t), t\right)= & \psi_{y}^{B} \hat{x}-\psi_{x}^{B} \hat{y} \\
= & \mathbf{u}^{B}(\mathbf{X}(t))+\left((i \hat{x}-\hat{y}) \sum_{n=2}^{\infty} n a_{n}(\epsilon r)^{n-1}\right. \\
& \left.\times e^{i(n-1) \theta}+\text { c.c. }\right)
\end{aligned}
$$

where $\hat{x}, \hat{y}$ are the unit vectors in the $x, y$ directions, respectively. We also expand the monopole vorticity in a Fourier series,

$$
\omega\left(\mathbf{x}^{\prime}+\mathbf{X}(t), t\right)=\omega_{0}(r, t)+\left(\sum_{n=1}^{\infty} \omega_{n}(r, t) e^{i n \theta}+\text { c.c. }\right)
$$

Since the coordinate system is centered at the vorticity centroid, the $n=1$ expansion coefficient of the vorticity will satisfy, ${ }^{13} \int_{0}^{\infty} r^{2} \omega_{1} d r=0$.

Substituting Eqs. (13) and (14) into (11), gives an expression for the velocity, $\mathbf{V}$, of the monopole,

$$
\mathbf{V}=\mathbf{u}^{B}(\mathbf{X}(t), t)+[(i \hat{x}+\hat{y}) \tilde{v}+\text { c.c. }],
$$

where $\tilde{v}$ is given by

$$
\tilde{v}=-\frac{2 \pi}{\Gamma} \sum_{n=3}^{\infty} n a_{n} \epsilon^{n-1} \int_{0}^{\infty} r^{n} \omega_{n-1} d r
$$

The monopole propagates at the velocity of the irrotational background flow evaluated at the vorticity centroid with a correction term that depends upon nonuniformities in the flow and the distortion of the monopole. We will be interested in irrotational flows that perturb the monopole slightly: these can be categorized as slowly varying flows corresponding to $\epsilon \ll 1,\left|a_{n}\right| \sim \mathcal{C}(1)$ and weak flows corresponding to $\epsilon=1,\left|a_{n}\right| \ll 1$. For either of these situations, the corrections to the monopole velocity are higher order. In Sec. III, we show the nonaxisymmetric distortion of the monopole is $\mathscr{C}(\epsilon)$ for a slowly varying flow and $Q\left(\left|a_{n}\right|\right)$ for a weak flow. Using this result in Eq. (16), the corrections to the vortex velocity for

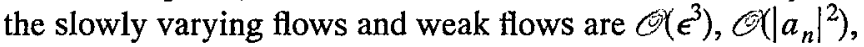
respectively.

A relation governing the centroid velocity of a monopole in an irrotational flow has been established by defining the vorticity centroid of the monopole and then applying the conservation of the first moment of vorticity. The leadingorder term in the velocity expansion agrees with the "coarse grained" vortex velocity obtained by Ting and Tung. ${ }^{19}$ They also calculated a "fine grained" solution which allows for the monopole velocity to oscillate about the centroid velocity. These "fine grained" velocity oscillations result from working in a coordinate system whose origin is displaced from the vorticity centroid. Viewed from the laboratory reference frame, a point off center will travel at the centroid 
velocity superposed with a rotational velocity about the centroid. We come back to this point in the discussion section of this paper where we explicitly show that the "fine grained" oscillations are removed by shifting the origin of the coordinate system to the vorticity centroid.

\section{Inhomogeneous equation for a monopole in an external flow}

In this subsection we transform the vorticity equation (1) into a reference frame that translates with the vorticity centroid, $\mathbf{X}(t)$. This yields an alternate form of the vorticity equation in which the effect of the external flow is represented as an inhomogeneous term.

Let the centroid of the vortex be located at $\mathbf{X}(t)$. A coordinate system relative to the centroid is given by

$$
\mathbf{x}^{\prime}=\mathbf{x}-\mathbf{X}(t), \quad t^{\prime}=t .
$$

The derivative operators transform according to the chain rule,

$$
\partial_{x_{i}}=\partial_{x_{i}^{\prime}}, \quad \partial_{t}=\partial_{t^{\prime}}-V_{i} \partial_{x_{i}^{\prime}}
$$

Note that the vorticity is invariant under this change of coordinates. Equations (1) and (2) become,

$$
\begin{aligned}
& \omega_{t}+\mathbf{u}^{\omega} \cdot \nabla \omega-\frac{1}{\operatorname{Re}} \nabla^{2} \omega=-\left(\mathbf{u}^{B}-\mathbf{V}\right) \cdot \nabla \omega, \\
& \nabla^{2} \psi^{\omega}=-\omega, \quad \mathbf{u}^{\omega}=\left(\psi_{y}^{\omega},-\psi_{x}^{\omega}\right),
\end{aligned}
$$

where the primes have been dropped from the independent variables and the velocity has been split into background and vorticity-induced components (i.e., $\mathbf{u}=\mathbf{u}^{\omega}+\mathbf{u}^{B}$ ). The initial profile of the vortex is specified by the initial condition, $\omega(\mathbf{x}, 0)$. Boundary conditions are imposed to require the velocity and vorticity to be continuous at the origin and decaying in the far field.

The right-hand side of Eq. (19) represents the distortion of the monopole streamlines by the external flow. For both the slow and weak external flows this term is small (i.e., $\left.\left|\mathbf{u}^{B}-\mathbf{V}\right| \ll 1\right)$ and represents a small perturbation to an axisymmetric monopole. In the next section a perturbation theory for the distortion of a monopole is developed in which the effect of the external flow enters as an inhomogeneous term.

\section{MONOPOLE DISTORTION IN AN EXTERNAL IRROTATIONAL FLOW}

In this section we will construct asymptotic solutions to Eq. (19) valid in the large Reynolds number limit. First, we describe the decay of an initially perturbed monopole in the absence of an external flow (i.e., $\mathbf{u}^{B}=\mathbf{V}=0$ ). In Sec. III B the distortion of a monopole in a perturbing external flow [i.e., $\left|\mathbf{u}^{B}-\mathbf{V}\right| \sim Q(\gamma)$ where $\left.\gamma \ll 1\right]$ is computed.

\section{A. Shear-diffusion mechanism}

We have previously studied the large Reynolds number linear stability of a Lamb monopole based on the ideas of Lundgren $^{22}$ and Rhines and Young. ${ }^{23}$ The main result is the mixing hypothesis which we restate here: ${ }^{13}$
Mixing Hypothesis: If an axisymmetric Lamb monopole, $W(r, T)$, is subject to a linear perturbation, $\bar{\omega}$, with zero mean along streamlines (i.e., $\oint \tilde{\omega} d \theta=0)$ and which preserves the first moment of vorticity, this perturbation will decay on an $Q\left(\mathrm{Rc}^{1 / 3}\right)$ time scale.

The mixing hypothesis identifies vorticity perturbations which decay on an $\left(\operatorname{Re}^{1 / 3}\right)$ time scale as those which are orthogonal to both axisymmetric redistributions of vorticity, and infinitesimal translations of the monopole. Thus, an initially perturbed monopole will relax to an axisymmetric state centered at the vorticity centroid on an $\Theta\left(\mathrm{Re}^{1 / 3}\right)$ time scale, much faster than the $G(\operatorname{Re})$ time scale for the viscous decay of a monopole.

The mechanism leading to the rapid decay of nonaxisymmetric vorticity perturbations is known as the sheardiffusion mixing mechanism, familiar from the study of the mixing of passive scalars by flows with closed streamlines. ${ }^{23-27}$ Rhines and Young elucidated the relevant scalings for the mixing of a passive scalar and emphasized the importance of simultaneous shearing and cross-stream diffusion in homogenizing the passive scalar along the streamlines of the flow. According to the mixing hypothesis, this same mechanism applies to small vorticity perturbations to a Lamb monopole as well. The difficulty in verifying this result for vorticity is due to the coupling of the vorticity with the flow field of the monopole and has been discussed in previous research. ${ }^{13,22}$

The decay of a vorticity perturbation by the sheardiffusion mechanism is best illustrated by a contour plot of the vorticity at various times (see Bernoff and Lingevitch ${ }^{13}$ ). The initial vorticity perturbation is sheared by the differential rotation inside the Lamb monopole. The shearing generates large vorticity gradients in the radial direction which are smoothed by the radial diffusion of vorticity on the $\mathrm{Re}^{1 / 3}$ time scale. In Fig. 2, the real and imaginary phases of an initial nonaxisymmetric perturbation to a Lamb monopole are plotted versus time at a fixed radius. The oscillations in the vorticity are due to the advection of the vorticity perturbation around the streamlines on the turnover time of the monopole. The envelope for the decay of these oscillations scales like $\mathrm{Re}^{1 / 3}$ for large Reynolds numbers. In the next section, the response of a monopole to an external flow is described for which the solution shown in Fig. 2 corresponds to a Green's function.

Through the mixing mechanism, we have argued that nonaxisymmetric, linear vorticity perturbations which are orthogonal to the translational modes of an isolated axisymmetric monopole will decay on the $\mathrm{Re}^{1 / 3}$ time scale. This contrasts with the inviscid dynamics of a perturbed axisymmetric monopole which has been studied by Dritschel. ${ }^{17} \mathrm{He}$ showed that an inviscid axisymmetric monopole with a monotonic vorticity profile is Lyapunov stable. The Hamiltonian nature of the Euler equations suggests that a perturbed inviscid vortex will oscillate about its unperturbed state indefinitely. A large Reynolds number monopole, however, will relax to an axisymmetric state due to the shear-diffusion mixing mechanism on the $Q\left(\operatorname{Re}^{1 / 3}\right)$ time scale. 


\section{B. Perturbation theory for distortions to a monopole}

Consider a monopole in an external perturbing flow. We work in a polar coordinate system with origin at the vorticity centroid and propose a perturbation expansion for the vorticity and the streamfunction about a localized axisymmetric state,

$$
\begin{aligned}
& \omega(\mathbf{x}, t) \sim \ddot{W}(r, T)+\gamma \tilde{\omega}(\mathbf{x}, t)+\Theta\left(\gamma^{2}\right), \\
& \psi^{\omega}(\mathbf{x}, t) \sim \Psi(r, T)+\gamma \tilde{\psi}(\mathbf{x}, t)+\circlearrowleft\left(\gamma^{2}\right),
\end{aligned}
$$

where $W, \Psi$ are functions of the radial coordinate and the viscous time scale, $T=t / \mathrm{Re}$. The small parameter, $\gamma$, is the strength of the external flow nondimensionalized on the characteristic monopole circulation velocity (i.e., $\left.\gamma=\left|\mathbf{u}^{B}-\mathbf{V}\right| /\left|\mathbf{u}^{\omega}\right|\right)$. We consider external flows. which are small perturbations to an axisymmetric vortex. These are categorized as slow and weak external flows with corresponding streamfunctions, $\bar{\psi}_{s}$ and $\bar{\psi}_{W}$. They have Taylor expansions about the vorticity centroid,

$$
\begin{aligned}
& \bar{\psi}_{S}=\frac{1}{\gamma}\left(\sum_{n=2}^{\infty} a_{n}(\gamma r)^{n} e^{i n \theta}+\text { c.c. }\right)+C\left(\gamma^{3}\right), \\
& \bar{\psi}_{W}=\left(\sum_{n=2}^{\infty} a_{n} r^{n} e^{i n \theta}+\text { c.c. }\right)+Q\left(\gamma^{2}\right) .
\end{aligned}
$$

The streamfunctions are arbitrary up to a constant, $a_{0}$, which has been set equal to zero and the $n=1$ coefficient, $a_{1}$, vanishes due to the fact that we transformed into a coordinate system that translates with the centroid velocity. For the slow external flow, the coefficients, $a_{n}$, are order unity [i.e., $\left.\left|a_{n}\right| \sim Q(1)\right]$ and for the weak flow, $\left|a_{n}\right| \sim Q(\gamma)$. The error terms in Eqs. (23) and (24) represent the correction to the streamfunction due to higher-order corrections to vortex velocity $V$. The leading-order effect of the external flow is $Q(\gamma)$ for both the slow and weak streamfunctions. From Eqs. (23) and (24), we see that the general form of the external streamfunction is

$$
\bar{\psi}=\gamma \bar{\psi}^{1}+\varrho\left(\gamma^{2}\right)=\gamma \sum_{n=2}^{\infty} A_{n}(\mathbf{X}(t), t) r^{n} e^{i n \theta}+\varrho\left(\gamma^{2}\right)
$$

where $A_{n}$ are the Taylor expansion coefficients of the external flow rescaled to be order unity. For a slow external flow, only $A_{2} \neq 0$, while for the weak external flow, $A_{n}=a_{n} / \gamma$.

Substituting these expansions into Eq. (19) and grouping terms in powers of $\gamma$ gives at $Q(1)$,

$$
W_{T}=\mathscr{L}^{0} \mathrm{~W}
$$

where $\mathscr{L}^{0}$ is the axisymmetric Laplacian operator,

$$
\mathscr{E}^{0}=\partial_{r r}+\frac{1}{r} \partial_{r}=\frac{1}{r} \partial_{r} r \partial_{r} .
$$

At leading order the vortex is axisymmetric leading to a decoupling of the vorticity equation from the streamfunction.

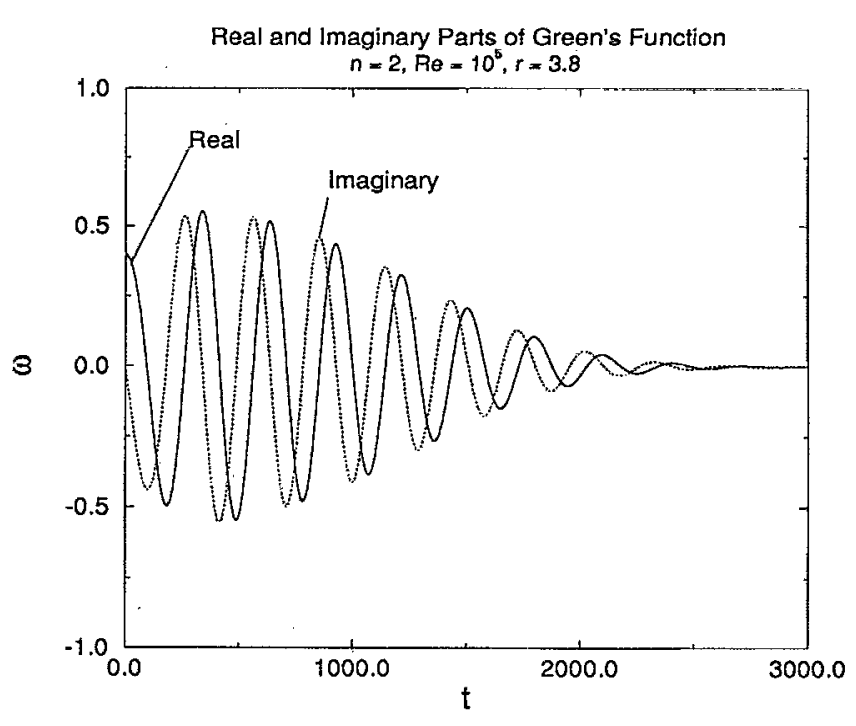

FIG. 2. The $n=2, \operatorname{Re}=10^{5}$ Green's function solution at a radius $r=3.8$ showing the decay of the real and imaginary componcnts of the vorticity perturbation with time. The oscillations in the Green's function are related to the turnover frequency of the Lamb monopole. The decay envelope for the oscillations scales as $\mathrm{Re}^{\mathrm{I} / 3}$, in agreement with the large Reynolds number asymptotic prediction.

The vorticity decays on the Reynolds number time scale due to viscous diffusion. The streamlines of flow are also axisymmetric and given by the solution of

$$
\mathscr{B}^{0} \Psi=-W
$$

The Lamb vortex specified in Eq. (3) is a particular axisymmetric solulion of Eqs. (26) and (28) corresponding to the self-similar viscous decay of an initial point vortex.

At $Q(\gamma)$ an inhomogeneous equation is obtained,

$\tilde{\omega}_{t}+\frac{1}{r} \partial_{\theta}\left(-\Psi_{r} \bar{\omega}+W_{r} \tilde{\psi}\right)-\operatorname{Re}^{-1} \nabla^{2} \ddot{\omega}=-\frac{1}{r} W_{r} \bar{\psi}_{\theta}^{1} \equiv I^{1}$,

$\nabla^{2} \tilde{\psi}=-\tilde{\omega}$,

where $\bar{\psi}^{1}$ is the $Q(\gamma)$ term in the Taylor expansion of the external flow. Physically the inhomogeneous term corresponds to the radial advection of the basic state vorticity, $W(r, T)$, by the external flow.

We proceed by expressing the vorticity and streamfunction perturbations, $(\tilde{\omega}, \tilde{\psi})$, in terms of their Fourier representations,

$$
\begin{gathered}
\tilde{\omega}(\mathbf{x}, t)=\omega_{0}(r, t)+\left(\sum_{n=1}^{\infty} \omega_{n}(r, t)+\text { c.c. }\right), \\
\tilde{\psi}(\mathbf{x}, t)=\psi_{0}(r, t)+\left(\sum_{n=1}^{\infty} \psi_{n}(r, t)+\text { c.c. }\right) .
\end{gathered}
$$

The equation for each Fourier component decouples, 


$$
\begin{aligned}
& \left(\omega_{n}\right)_{t}+i n \frac{1}{r}\left(-\Psi_{r} \omega_{n}+W_{r} \psi_{n}\right)-\operatorname{Re}^{-1} \mathscr{E}^{n} \omega_{n} \\
& =-i n \frac{1}{r} W_{r} A_{n} r^{n} \equiv I_{n}^{1}, \\
& \mathscr{E}^{n} \psi_{n}=-\omega_{n}, \\
& \mathscr{L}^{n}=\frac{1}{r} \partial_{r} r \partial_{r}-\frac{n^{2}}{r^{2}},
\end{aligned}
$$

where the amplitudes $A_{n}$ are the $(\gamma)$ Taylor expansion coefficients of the external flow and depend upon the centroid location and time. The velocity and vorticity are required to be continuous at the origin,

$$
\psi_{n}, \omega_{n} \propto r^{n} \quad \text { as } r \rightarrow 0 .
$$

Exponential localization of the monopole leads to

$$
\left(\psi_{n}\right)_{r}, \omega_{n} \rightarrow 0 \text { as } r \rightarrow \infty \text {. }
$$

For $n=0,1$, the inhomogeneous term vanishes as can be seen from Eq. (25). Thus, at $O(\gamma)$, the external flow does not radially redistribute vorticity nor does it translate the monopole. For $n \geqslant 2$ the inhomogeneous term creates a nonaxisymmetric distortion of the monopole.

The solution of Eq. (31) can be approximated in the limit of large Reynolds number in terms of a convolution integral with a Green's function. The linear operator on the left-hand side of Eq. (31) depends on time through the coefficients $W(r, T), \Psi(r, T)$. These coefficients evolve on the Reynolds number time scale, much slower than the shear-diffusion time scale [i.e., $\left.\bigodot\left(\operatorname{Re}^{1 / 3}\right)\right]$. Treating the $T$ dependence of the coefficients as an independent parameter, we define the Green's function as the solution of

$$
\begin{aligned}
G_{t}^{n} & +i n \frac{1}{r}\left(-\Psi_{r} G^{n}+W_{r} H^{n}\right)-\operatorname{Re}^{-1} \mathscr{S}^{n} G^{n} \\
& =-i \frac{W_{r}}{r} r^{n} \delta(t)
\end{aligned}
$$

$\mathscr{Z}^{n} H^{n}=-G^{n}$,

where $\delta(t)$ is the Dirac delta function in time; $G^{n}(r, t ; T)$, $H^{n}(r, t ; T)$ are the Green's functions for the vorticity and corresponding streamfunction, respectively. The Green's function is zero for $t<0$. Since all $n \geqslant 2$ perturbations satisfy the conditions of the mixing hypothesis, the Green's function is exponentially decaying on the shear-diffusion time scale for $t>0$ (see Fig. 2). The solution of Eq. (31) becomes a convolution integral,

$$
\omega_{n}(r, t)=\int_{0}^{\infty} A_{n}(\mathbf{X}(t-\tau), t-\tau) G^{n}(r, \tau ; T) d \tau .
$$

Utilizing this solution, we can estimate the response of the monopole to a time-dependent external flow.

If the time scale of the external flow is much slower than the shear-diffusion time scale (i.e., $T_{e} \gg \mathrm{Re}^{1 / 3}$ ), then the monopole relaxes to a quasisteady state,

$$
\omega_{n}(r, t)=A_{n}(\mathbf{X}(t), t) \int_{0}^{\infty} G^{n}(r, \tau ; T) d \tau
$$

The monopole equilibrates to this state via the sheardiffusion mixing mechanism on the $\mathrm{Re}^{1 / 3}$ time scale. From Eq. (31), we see that at leading order, the quasisteady statc satisfies an ordinary differential equation for the perturbation streamfunction,

$$
\mathscr{L}^{n} \psi_{n}+\frac{W_{r}}{\Psi_{r}} \psi_{n}=-\frac{W_{r}}{\Psi_{r}} A_{n}(\mathbf{X}(t), t) r^{n}
$$

This agrees with the "coarse grained" solution obtained by Ting and his collaborators. ${ }^{19,20}$ They interpreted the "coarse grained" solution as the time average evolution of the monopole. In addition, they computed a "fine grained" solution which includes oscillations in the vorticity on the monopole turnover time. Their description, however, overlooks the shear-diffusion mechanism which attenuates these fine scale oscillations on a $\mathrm{Re}^{1 / 3}$ time scale, thus, allowing the monopole to relax to a quasisteady state.

If the time scale of the external flow is not much slower than the shear-diffusion time scale, the linear response of the monopole is bounded by

$$
\left|\omega_{n}(r, t)\right| \leqslant\left|\sup _{t^{\prime} \leqslant t} A_{n}\left(\mathbf{X}\left(t^{\prime}\right), t^{\prime}\right)\right| \int_{0}^{\infty}\left|G^{n}(r, \tau ; T)\right| d \tau .
$$

The Green's function decays on the shear-diffusion time scale and a Wentzel-Kramers-Brillouin (WKB) analysis ${ }^{13}$ shows

$$
G^{n}(r, \tau) \leqslant C \exp \left[i n \Omega t-n^{2}\left(\Omega_{r}\right)^{2} t^{3} /(3 \mathrm{Re})\right],
$$

for large times where $\Omega=-\Psi_{r} / r$ is the angular velocity of the base state monopole and $C$ is a constant. From this estimate, a bound on the response of the monopole becomes

$$
\left|\omega_{n}(r, t)\right| \leqslant \bar{C} \operatorname{Re}^{1 / 3} \frac{1}{\left(n \Omega_{r}\right)^{2 / 3}} \mid \begin{aligned}
& \sup _{t^{\prime} \leqslant t} A_{n}\left(\mathbf{X}\left(t^{\prime}\right), t^{\prime}\right) \\
& \mid
\end{aligned}
$$

where $\bar{C}$ is a constant. This allows for the possibility of a large amplitude, $G\left(\mathrm{Re}^{1 / 3}\right)$, resonant response. In the next section, we solve Eq. (31) numerically and show that the response of the monopole is $\Theta\left(\mathrm{Re}^{1 / 3}\right)$ when the external flow is in resonance with the monopole whereas in the nonresonant case the response of the monopole is order unity.

The $Q(\gamma)$ perturbation theory is valid up to the time at which the error due to ignoring the effect of the nonlinear term in the vorticity equation becomes $O(1)$. We estimate this time by considering the $Q\left(\gamma^{2}\right)$ terms in the perturbation theory. At this order, the nonlinear interaction of the vorticity perturbation, $\bar{\omega}$, with its induced streamfunction, $\tilde{\psi}$, contributes to the inhomogeneous term. In the nonresonant case [i.e., $\tilde{\omega} \sim \mathcal{Q ( 1 )}$ ], the nonlinear term will lead to an $\Theta\left(\gamma^{2}\right)$ correction to the velocity of the monopole and an axisymmetric redistribution of vorticity. These errors will accumulate on an $Q\left(1 / \gamma^{2}\right)$ time scale; requiring this nonlinear time scale to be much longer than the viscous decay time of the monopole yields, $\gamma \leqslant \operatorname{Re}^{-1 / 2}$. In the resonant case (i.e., $\tilde{\omega} \sim \mathrm{Re}^{1 / 3}$ ), an analogous estimate for the effect of the nonlinear term yields $\gamma<\mathrm{Re}^{-5 / 6}$

In this section, we have described the distortion of a monopole in a perturbing irrotational flow at large Reynolds 
numbers. We have shown that the distortion of the monopole is given in terms of a convolution integral of a Green's function with an inhomogeneous forcing. Due to a sheardiffusion mixing mechanism, the Green's function decays exponentially on the $\operatorname{Re}^{1 / 3}$ time scale. Based on this description, we find that for slowly varying time-dependent flows, the monopole relaxes to a quasisteady state on the $\mathrm{Re}^{1 / 3}$ time scale. For general time-dependent flows, we have approximated a bound on the response amplitude and described the possibility of a resonant interaction between the monopole and the external flow. The amplitude of the resonant response scales as $\operatorname{Re}^{1 / 3}$ due to a balance between energy dissipation by the shear-diffusion mechanism and energy input from the external flow. In the next section, we numerically solve the vorticity equation linearized about a Lamb monopole to verify the results of this asymptotic theory.

\section{NUMERICAL VERIFICATION OF ASYMPTOTIC THEORY}

We consider as our canonical problem, a Lamb monopole centered in a straining flow. As can be seen from Eq. (23), a straining flow is the leading-order term in the Taylor expansion of a slow external flow,

$$
\bar{\psi}^{1}\left(\mathbf{x}^{\prime}, t\right)=A_{2} r^{2} e^{i 2 \theta}+\text { c.c. }
$$

where $A_{2}$ is the coefficient of the quadratic term.

In this section, we numerically computc the frequency response of the monopole and show that the amplitude of the response is $\mathscr{Q}\left(\mathrm{Re}^{1 / 3}\right)$ when the the external flow is resonant with the monopole. For the numerical results of this section a time-periodic straining flow is chosen with $A_{2}$ given by

$$
A_{2}=i e^{-i \Omega_{F} t} \text {. }
$$

This corresponds to a stagnation point flow rotating in the counterclockwise direction with rotational frequency, $\Omega_{F} / 2$. With this choice of external flow, the linearized vorticity equation becomes,

$$
\begin{aligned}
& \left(\omega_{2}\right)_{t}+i 2 \frac{1}{r}\left(-\Psi_{r} \omega_{2}+W_{r} \psi_{2}\right)-\operatorname{Re}^{-1} \mathscr{E}^{2} \omega_{2} \\
& =2 r^{2} \frac{1}{r} W_{r} e^{-i \Omega_{F} t}, \\
& \mathscr{E}^{2} \psi_{2}=-\omega_{2} .
\end{aligned}
$$

The harmonic time dependence can be separated from the rest of the perturbation by the transformation,

$$
\omega_{2}=\hat{\omega} e^{-i \Omega_{F} t}, \quad \psi_{2}=\hat{\psi} e^{-i \Omega_{F} t},
$$

yielding,

$$
\begin{aligned}
\hat{\omega}_{t}+i & {\left[2 \Omega(r)-\Omega_{r}\right] \hat{\omega}+i 2 \frac{W_{r}}{r} \hat{\psi}-\operatorname{Re}^{-1} \mathscr{L}^{2} \hat{\omega} } \\
& =2 r^{2} \frac{1}{r} W_{r},
\end{aligned}
$$

$\mathscr{L}^{2} \hat{\psi}=-\hat{\omega}$,

where $\Omega(r)=-\Psi_{r} / r$ is the angular velocity of the base state vorticity distribution. From Eq. (46), we see that the time dependence of the external flow effectively reduces the angular velocity of the base flow by a constant.

The base state monopole that we choose for our numerical experiments is a Lamb monopole [see Eq. (3)] with $T=1$. The viscous decay of the base state on the Reynolds number time scale has been suppressed. We will see that this approximation is valid in the large Reynolds number limit since the monopole relaxes to periodic oscillations much more rapidly than the Reynolds number decay time scale of the monopole.

Equation (46) is solved using a second-order split-step method. The streamfunction and vorticity perturbation are discretized in the radial direction using second-order centered finite differences. The diffusion step is implemented implicitly using Crank-Nicholson and the advection step cxplicitly with fourth-order Runge-Kutta; the perturbation streamfunction at each time step is computed as a tridiagonal inversion of the Laplacian operator.

The frequency response of the Lamb monopole is shown in Fig. 3 as a function Reynolds number. Figure 3(a) is a plot the maximum vorticity amplitude and Fig. $3\left(\right.$ b) is the $L_{2}$ norm of the vorticity perturbation. The response curves exhibit a resonant peak at which the energy input from the external flow is balanced by the dissipation due to the sheardiffusion mechanism. The scaling of the peak response amplitude with Reynolds number is approximated by a local analysis of Eq. (46) about the resonant streamline at $r=R$. Assuming that the vorticity behaves as a passive scalar and seeking a steady solution yields,

$$
i \alpha \rho \hat{\omega}-\operatorname{Re}^{-1} \hat{\omega}_{p p}=\beta,
$$

where $\rho$ is a local radial coordinate (i.e., $r=R+\rho$ ) and,

$$
\alpha=\left.2 \frac{d \Omega}{d r}\right|_{r=R}, \quad \beta=\left.2 r W_{r}\right|_{r=R} .
$$

In Eq. (47), the streamfunction-vorticity coupling has been suppressed and only the leading-order diffusion term $\hat{\omega}_{\rho \rho}$ is retained. Rescaling $\rho$ and $\hat{\omega}$ to balance the effects of shear, diffusion, and the external forcing yields a similarity solution,

$$
\hat{\omega}=\operatorname{Re}^{1 / 3} \frac{\beta}{\alpha^{2 / 3}} f\left(\alpha \operatorname{Re} \rho^{3}\right) .
$$

From this, we see that the maximum vorticity amplitude at the resonant streamline scales as $\mathcal{C}\left(\operatorname{Re}^{1 / 3}\right)$ and the response is localized about the streamline with length scale of O $\left(\operatorname{Re}^{-1 / 3}\right)$.

Figure 4(a) is a $\log -\log$ plot of the maximum perturbation amplitude and $L_{2}$ perturbation norm versus Reynolds number. The maximum vorticity amplitude is in good agreement with the $\operatorname{Re}^{1 / 3}$ asymptotic prediction for large Reynolds numbers. This supports the argument that the dissipative shear-diffusion mechanism is balancing energy input from the external flow. The scaling of the $L_{2}$ perturbation norm with Rcynolds number yields a slope of 0.150 which differs slightly from the theoretical slope of $1 / 6$. We attribute the slow convergence of the $L_{2}$ norm to its asymptotic value as due to the order unity contributions to the vorticity away from the resonant streamline. Figure $4(\mathrm{~b})$ is a plot of the forcing frequency at which the response amplitude is maxi- 
Maximum Vorticity Amplitude vs. Forcing Frequency

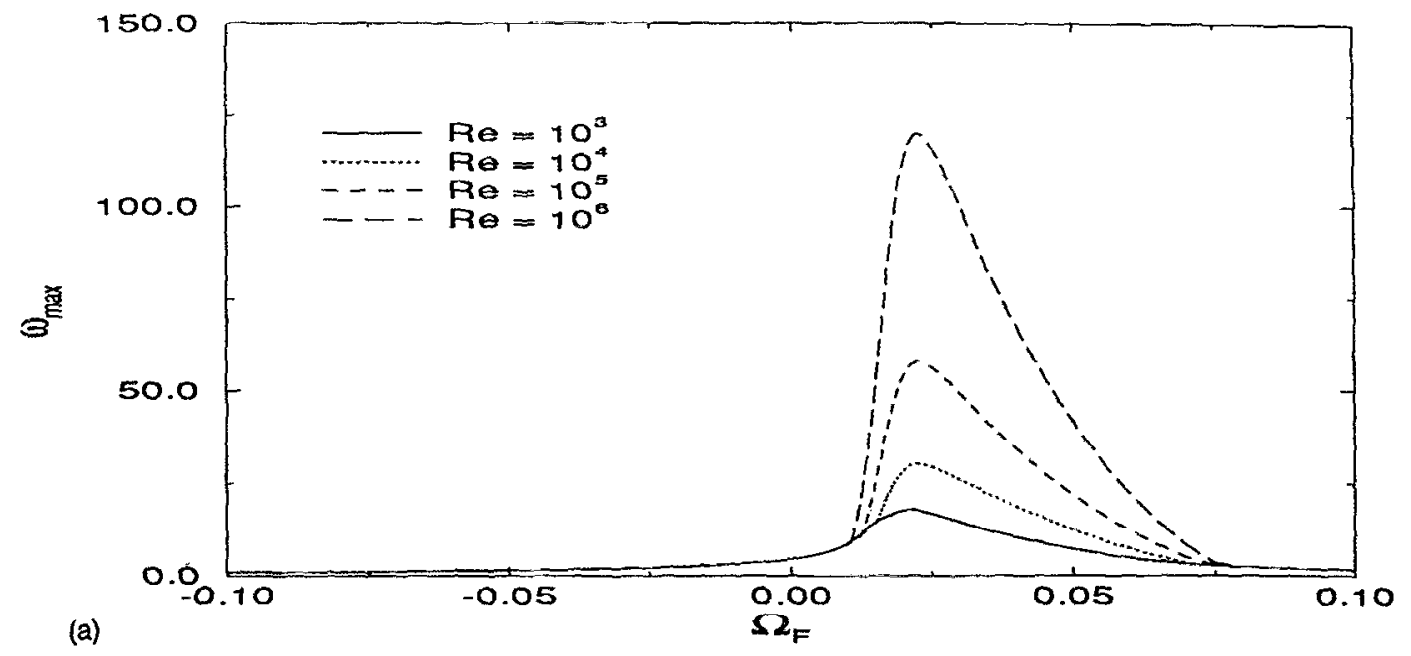

$L_{2}$ Norm ve. Forcing Frequency

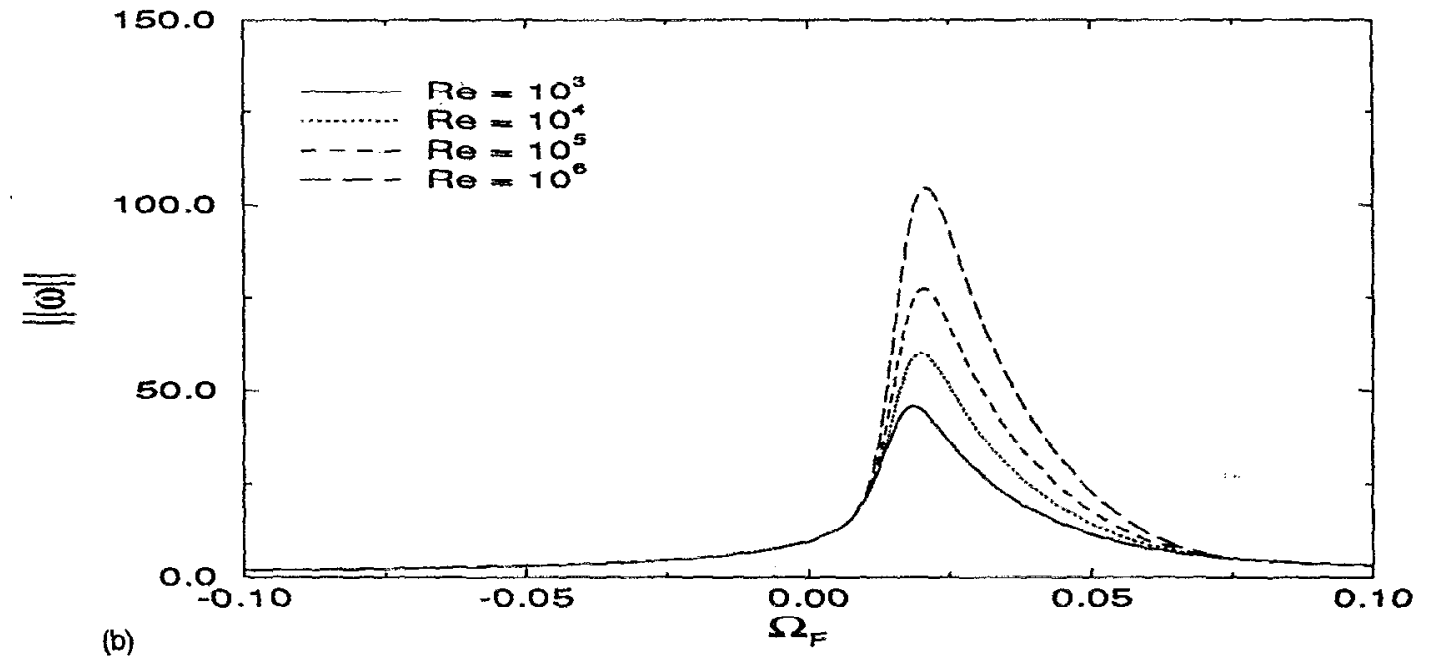

FIG. 3. Frequency response of a Lamb monopole to a time-periodic straining flow $(n=2)$. Panel (a) shows the maximum of the vorticity response, $\omega_{\max }$, versus forcing frequency, $\Omega_{F}$, and (b) shows the $L_{2}$ norm of the vorticity perturbation, $\|\omega\|$ versus forcing frequency for $\operatorname{Re}=10^{3}, 10^{4}, 10^{5}, 10^{6}$. The peak in the response curves are due to the resonant forcing of the monopole at twice the turnover frequency, $\Omega_{F}=2 \Omega(r)$. At resonance, dissipation of the shear-diffusion mechanism balances the energy input from the external flow.

mized versus Reynolds number. The frequency asymptotes to a constant value of $\Omega_{F}^{*} \approx 0.0225$ as the Reynolds number becomes large. This differs significantly from the predicted value, 0.0482, from Eq. (49) which neglects the streamfunction-vorticity coupling.

The radial position of the maximum amplitude response for a passive scalar and vorticity are plotted versus forcing frequency in Fig. 5. The passive scalar data corresponds to a time-periodic source released into the flow of a Lamb monopole. For a range of frequencies, the maximum response occurs at a radius given by $\Omega_{F}=2 \Omega(r)$ which corresponds to the resonant excitation of a particular streamline of the monopole. As the frequency of the external forcing is decreased, the resonant streamline moves radially outward and finally into the region of exponentially small vorticity. Increasing the frequency shifts the maximum amplitude vorticity radially inward until the external frequency exceeds the maximum turnover frequency of the monopole.

Figure 6 is a plot of the real and imaginary parts of the vorticity at a forcing frequency of $\Omega_{F}=0.02125$ and * Reynolds numbers $10^{4}, 10^{5}, 10^{6}$ showing the localization of the vorticity about the resonant streamline. The monopole relaxes to this state on the $\mathrm{Re}^{1 / 3}$ time scale. Recall that the time periodicity of the perturbation has been explicitly removed by the transformation in Eq. (45), so, Fig. 6 represents the Fourier amplitude of the periodic response of the monopole. Figure 7 compares the frequency response of a passive scalar and vorticity. There is excellent agreement between the pas- 

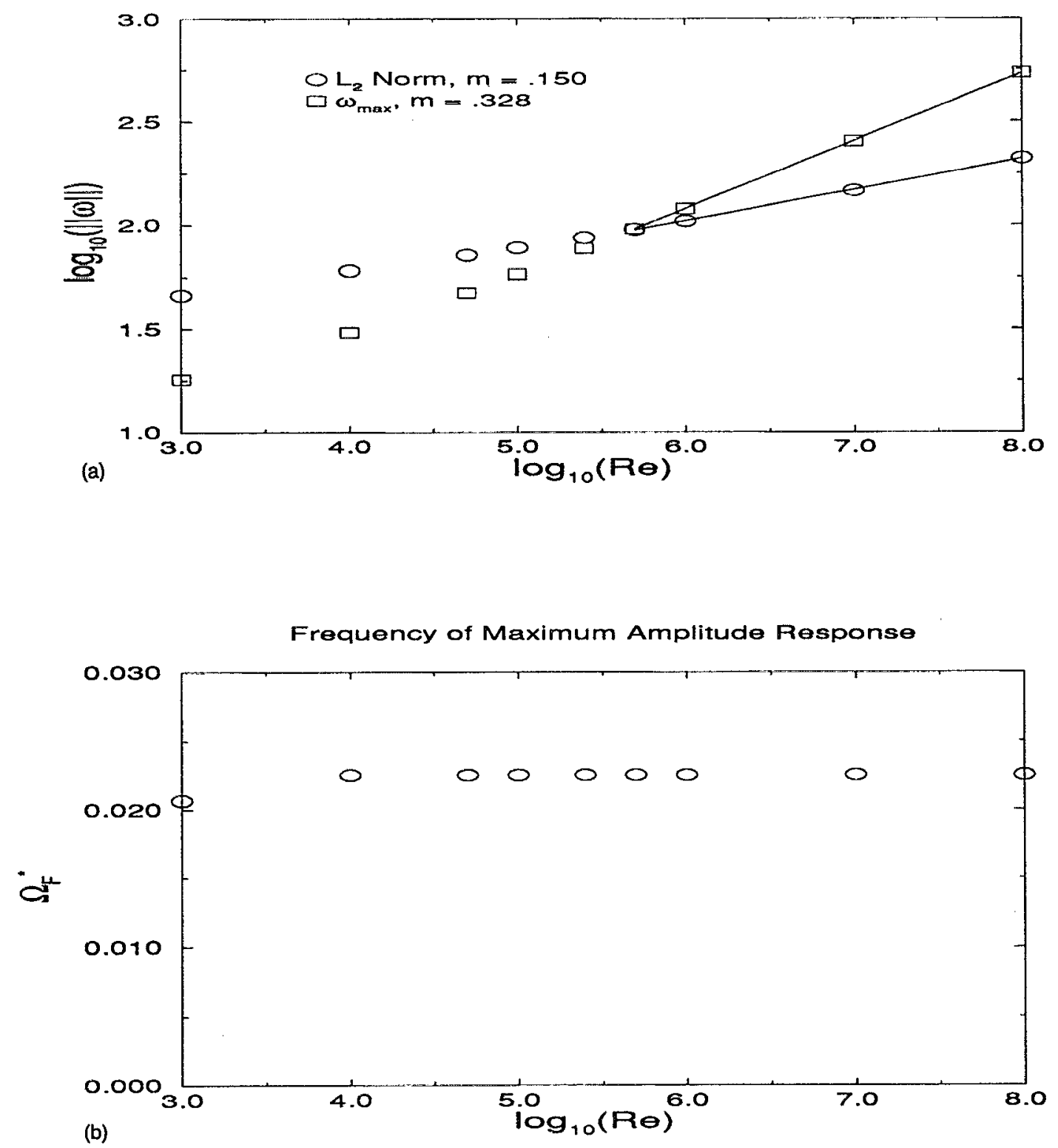

FIG. 4. Panel (a) is a $\log -\log$ plot of the maximum vorticity response versus Reynolds number for a Lamb monopole in a time-periodic external straining fow. The data are in agreement with the predicted asymptotic slopes of $1 / 3$ for the maximum vorticity and $1 / 6$ for the $L_{2}$ norm of the vorticity response. Panel (b) is a $\log$ plot of the frequency of the maximum amplitude response, $\Omega_{F}^{*}$, versus Reynolds number. The frequency of the maximum response approaches a constant value $\Omega_{F}^{*} \sim 0.02250$ at large Reynolds numbers.

sive scalar data and the asymptotic theory, which verifies our analysis. The frequency response of the vorticity, however, is shifted to a lower frequency due to the streamfunctionvorticity coupling.

The numerical solutions discussed above support the large Reynolds number asymptotics of Sec. III. We have shown that due to the shear-diffusion mechanism, a monopole in an external flow equilibrates to a periodically distorting shape on the $\mathrm{Re}^{1 / 3}$ time scale. The amplitude of the periodic distortion is order unity for most frequencies but there is a resonant range when the response scales as $\operatorname{Re}^{1 / 3}$. The amplitude of the resonant response is explained by a balance of energy into the monopole from the external flow and dissipation by the shear-diffusion mechanism.

\section{DISCUSSION}

In this section, we attempt to elucidate the relationship between the asymptotic results of this paper and previous work in the field. Ting et al. ${ }^{19,20}$ and Gunzburger ${ }^{21}$ have considered essentially the same problem as is discussed herein. They described the viscous decay of a monopole in terms of a multiple time scale asymptotic solution where the relevant time scales were the monopole turnover time and the Reynolds number time scale (i.e., an inviscid time scale and a viscous time scale). In our work, we include the sheardiffusion time scale which is intermediate between the inviscid and viscous time scales. An additional difference between our work and theirs is the choice of coordinate system. 
Radial Position of Maximum Amplitude Response vs Frequency

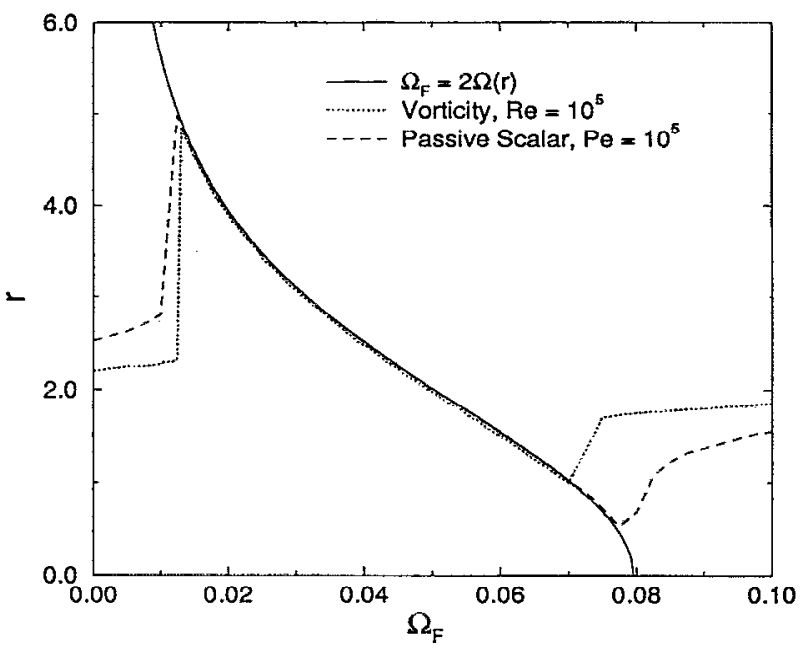

FIG. 5. Radial position of the maximum amplitude response versus forcing frequency for: (1) a time-periodic, $n=2$ source of passive scalar mixed in the flow field of a Lamb monopole and (2) the vorticity perturbation of a Lamb monopole in a time-periodic straining flow. At resonance, the maximum amplitude response occurs at the streamline for which $\Omega_{F}=2 \Omega(r)$.

We choose the vorticity centroid of the monopole as the origin of our coordinate system. They choose the center of the leading-order axisymmetric solution as their origin, which can differ from the centroid by a distance of order $\mathrm{Re}^{-1 / 2}$ due to their first-order correction. This difference manifests itself in two ways in their expansion; the appearance of the translational mode at first order and the rotation of the origin about the centroid.

To see this difference explicitly we examine the firstorder distortion of the monopole vorticity, $\bar{\zeta}^{(1)}$, from Gunzburger (setting his vortex strength $\Gamma$ and initial time $T_{0}$ to unity to agree with our nondimensionalization and using our nomenclature),

$$
\bar{\zeta}^{(1)}=-\frac{2 \hat{U}_{0}}{T^{3 / 2}}\left[\eta \exp \left(-\eta^{2}\right)\right] \sin \left(\theta-p_{0}-\frac{\operatorname{Re}}{8 \pi} \ln (T)\right),
$$

where $\hat{U}_{0}$ is the speed of the monopole relative to the background flow, $T$ is the viscous time, and $\eta=r / \sqrt{4 T}$. Equation (50) is precisely a multiple of the translational mode for the Lamb vortex, ${ }^{13}$

$$
\bar{\zeta}_{\text {trans }}^{(1)}=\mathbf{x}_{0} \cdot \nabla W=-\frac{r}{8 \pi T^{2}} e^{-r^{2} /(4 T)} \mathbf{x}_{0} \cdot \hat{r} .
$$

Ting et al. and Gunzburger allow the initial velocity of the vorlex to be specified at order $\mathrm{Re}^{-1 / 2}$; this can be accommodated by choosing the origin slightly shifted from the centroid. The amplitude of the translational mode in (50) is proportional to the relative speed of the monopole, $\hat{U}_{0}$, and follows from a small $r$ Taylor expansion of the velocity field due to a Lamb monopole. Using Eq. (3), an expression relating the displacement of the origin, $\left|\mathbf{x}_{0}\right|$, to the relative speed of the monopole is obtained,

$$
\hat{U}_{0}=\frac{\left|\mathbf{x}_{0}\right|}{8 \pi}+\cdots .
$$

Note that if the relative speed $\hat{U}_{0}$ vanishes, the origin coincides with the vorticity centroid; this is the coordinate system we have chosen. The angular velocity of a point shifted off the vortex centroid also follows from the first term in the Taylor expansion for the angular velocity,

$$
\frac{d \theta}{d T}=-\frac{\operatorname{Re}}{8 \pi T}, \quad \theta(T)=\theta_{0}-\frac{\operatorname{Re}}{8 \pi} \ln (T) .
$$

This shows that the time-dependent phase in Eq. (50) is due to the rotation of the displaced center about the centroid. Note that the angular velocity decreases on the viscous time scale as the Lamb monopole decays. In summary, this oscillation can be removed by choosing a coordinate system whose origin coincides with the centroid.

By including the shear-diffusion mechanism into the analysis of a decaying vortex, we have described how an initially perturbed vortex equilibrates in an external flow. Ting $e t$ al. and Gunzburger average over non-null perturbations of the monopole to yield a time-averaged description of a distorted monopole. Although the Lamb monopole is a stable solution of the Euler equations, ${ }^{11}$ they do not address the large Reynolds number stability of the monopole. Previously we have shown ${ }^{13}$ that the Lamb monopole is stable and nonaxisymmetric disturbances decay due to the sheardiffusion mechanism on the $\mathrm{Re}^{1 / 3}$ time scale.

Finally, we have examined the distortion and evolution of the monopole due to an external straining flow. The quasisteady quadrupolar distortion of the Lamb monopole derived here is in agreement with the previous work of Ting et $a l^{19,20}$ We extend this analysis to consider time-dependent straining flows which vary on the monopole turnover time.

A qualitative comparison of these results with elliptical inviscid models can be made. We first note that for weak straining flows circular patches become elliptical, ${ }^{14,15}$ which is effectively a quadrupolar distortion. This elliptical distortion is also observed by Dritschel and Legras ${ }^{17,18}$ for monotonic vorticity distributions in sufficiently weak straining flows. The stripping of vorticity for stronger straining flows is a fully nonlinear effect and is beyond the asymptotic methods presented here. Dritschel ${ }^{16}$ identified a resonant mechanism of instability whereby oscillations of a patch in phase with the rotational frequency of the patch relative to the straining field are amplified; this mechanism is similar to the resonant amplification mechanism described herein.

\section{CONCLUSIONS}

In this paper we have studied the interaction of a localized vortex monopole with an external irrotational flow at large Reynolds number. This work builds upon the previous research of Ting and his collaborators, and Gunzburger who studied the viscous regularization of a point vortcx in an external flow. ${ }^{19-21}$

The monopole is advected at a velocity which is determined from the conservation of the first moment of vorticity, a quantity analogous to momentum. To leading order this yields that the monopole propagates at the velocity of the 


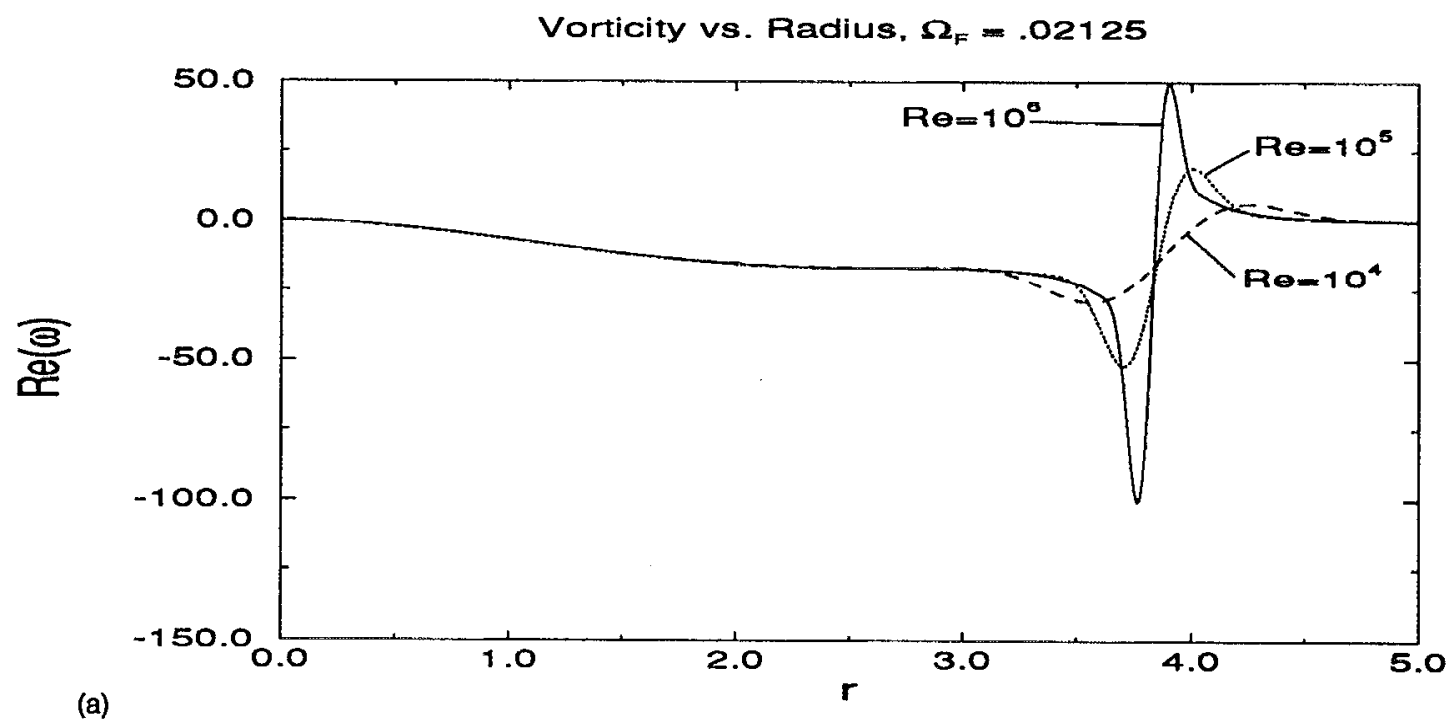

(a)

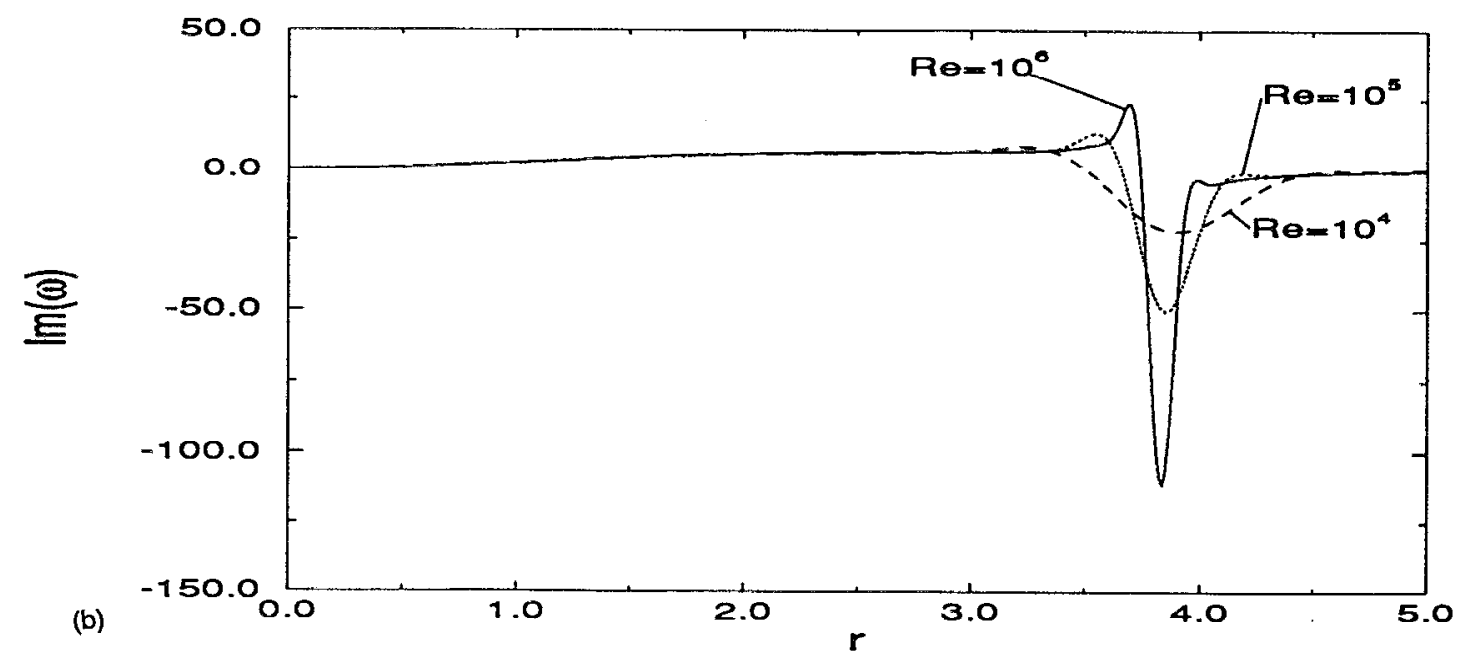

FIGG. 6. The real and imaginary parts of the vorticity versus radius for a Lamb monopole in a time-periodic straining flow (the oscillatory time-dependent factor has been factored out). The forcing frequency is $\Omega_{F}=0.02125$ and the maximum in the vorticity occurs at $r \approx 3.8$. Note as the Reynolds number is increased the amplitude of the maximum response scales likc $\mathrm{Re}^{1 / 3}$ and the width of the peak like $\mathrm{Re}^{-1 / 3}$.

irrotational flow evaluated at the vorticity centroid in agreement with the previous work of Ting and Tung.

Nonuniformities in the external flow act to distort the vorticity distribution of the monopole. A new facet of this research is that we have included the effects of the sheardiffusion mechanism into a large Reynolds number asymptotic theory for the evolution of the monopole. Through this mechanism, nonaxisymmetric distortions of the monopole are equilibrated on an $Q\left(\operatorname{Re}^{1 / 3}\right)$ shear-diffusion time scale which is much faster than the viscous decay time, $Q(\mathrm{Re})$, of the monopole.

We have verified the asymptotic results through a numerical solution of the linearized equations for the canonical example of a Lamb monopole in a time-periodic straining flow. The numerical results support the rapid relaxation of the monopole to a quasisteady state for slowly varying external flows and periodic oscillations for time harmonic external flows. We have also computed the frequency response of the monopole, showing that a resonant forcing of the monopole leads to a large response near the streamline that is in resonance with the external forcing. The amplitude of the vorticity perturbation maximum scales as $\mathrm{Re}^{1 / 3}$ in agreement with a local asymptotic analysis which treats the vorticity perturbation as a passive scalar. The analogy between a passive scalar and vorticity is limited, however, because the streamfunction-vorticity coupling shifts the maximum of the frequency response.

A large amplitude resonant response limits the validity 
Comparison of Maximum Response for Passive Scalar and Vorticity

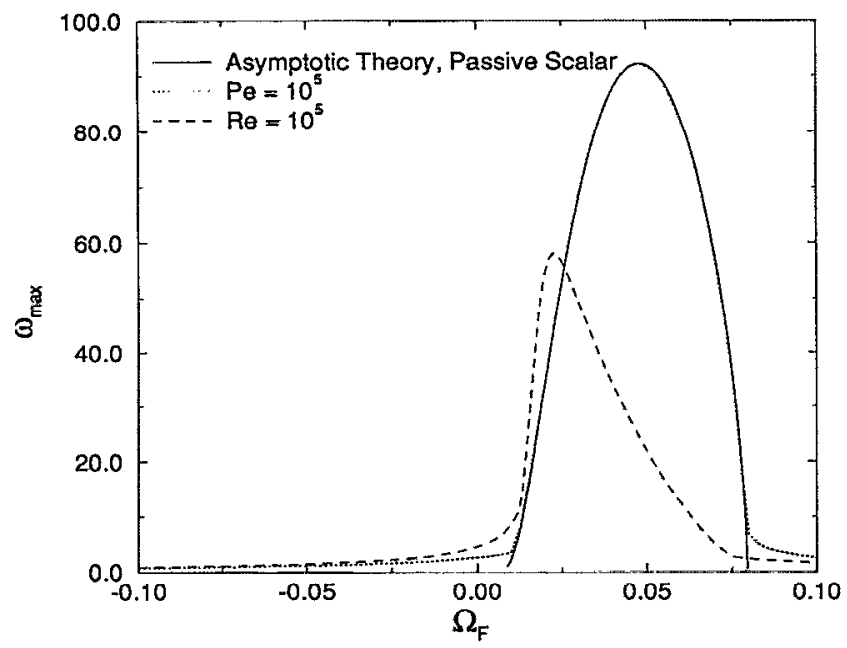

FIG. 7. Comparison of the frequency response of a time-periodic source of passive scalar mixed in the flow field of a Lamb monopole and a vorticity perturbation to a Lamb monopole in a time-periodic straining flow. The shape of the passive scalar response curve is in excellent agreement with that predicted by asymptotic theory. The difference of the vorticity response curve is due to the streamfunction-vorticity coupling.

of our small amplitude theory and requires further study. Currently, we are collaborating with Dr. Louis Rossi on fully nonlinear numerical simulations of a monopole in a resonant straining flow. One of the problems suggested by this research will be to determine the fate of the monopole as the external flow amplitude is increased beyond the linear regime.

\section{ACKNOWLEDGMENTS}

This work has been supported through NSF Grant No. CTS-9206828. We thank Louis Rossi for his helpful discussions and comments on this manuscript. We also wish to thank the referees for their constructive comments. One of us (AJB) wishes to thank "The Tribe" for inspiration and perspiration (in the sense of Edison). The other (JFL) wishes RJL a zeroth HBD.

${ }^{1} \mathrm{~K}$. Ahlnas, T. C. Royer, and T. H. George, "Multiple dipole eddies in the Alaska Coastal Current detected with Landsat thematic mapper data," J. Geophys. Res. 92, 13041 (1987).

${ }^{2}$ Y. Couder and C. Basdevant, "Experimental and numerical study of vortex couples in two-dimensional flows," J. Fluid Mech. 173, 225 (1986).

${ }^{3}$ G. J. F. VanHeist and J. B. Flór, "Dipole formation and collisions in a stratified fluid," Nature 340, 212 (1989).

${ }^{4}$ A. D. Gilbert, "Spiral structures and spectra in two-dimensional turbulence," J. Fluid Mech. 193, 475 (1988).

${ }^{5} \mathrm{~S}$. Kida and K. Ohkilani, "Spatiotemporal intermittency and instability of a forced turbulence," Phys. Fluids A 4, 1018 (1992).

${ }^{6} \mathrm{~J}$. C. McWilliams, "The emergence of isolated coherent vortices in turbulent tlow," J. Fluid Mech. 146, 21 (1984).

${ }^{7}$ H. K. Moffatt, S. Kida, and K. Ohkitani, "Stretched vortices-The sinews of turbulence; Large-Reynolds-number asymptotics," J. Fluid Mech. 259, 241 (1994).

${ }^{8} \mathrm{G}$. K. Batchelor, An Introduction to Fluid Dynamics (Cambridge University Press, Cambridge, 1967).

${ }^{9}$ P. G. Saffman, Vortex Dynamics (Cambridge University Press, Cambridge, 1992).

${ }^{10} \mathrm{P}$. J. Thomas and D. Auerbach, "The observation of the simultaneous development of a long- and short-wave instability mode on a vortex pair," J. Fluid Mech. 265, 289 (1994).

${ }^{11}$ D. G. Dritschel, "Nonlincar stability bounds for inviscid two-dimensional, parallel or circular flows with monotonic vorticity, and the analogous quasi-geostrophic flows," J. Fluid Mech. 191, 575 (1988).

${ }^{12}$ M. V. Melander, J. C. McWilliams, and N. J. Zabusky, "Axisymmetrization and vorticity-gradient intensification of an isolated two-dimensional vortex through filamentation," J. Fluid Mech. 178, 137 (1987).

${ }^{13}$ A. J. Bernoff and J. F. Lingevitch, "Rapid relaxation of an axisymmetric vortex," Phys. Fluids 6, 3717 (1994).

${ }^{14} \mathrm{D}$. W. Moore and P. G. Saffman, "Structure of a line vortex in an imposed strain," in Aircraft Wake Turbulence, edited by J. Olsen, A. Goldberg, and N. Rogers (Plenum, New York, 1971), pp. 339-354.

${ }^{15} \mathrm{~S}$. Kida, "Motion of an elliptic vortex in a uniform shear flow," J. Phys. Soc. Jpn. 50, 3517 (1981).

${ }^{16} \mathrm{D}$. G. Dritschel, "The stability of elliptical vortices in an external straining flow," J. Fluid Mech. 210, 223 (1990).

${ }^{17}$ D. G. DritscheI, in Mathematical Aspects of Vortex Dynamics, edited by R. E. Caflisch (SIAM, Philadelphia, 1988).

${ }^{18} \mathrm{~B}$. Legras and D. G. Dritschel, "Vortex stripping and the generation of high vorticity gradients in two-dimensional flows," Appl. Sci. Res. 51, 445 (1993).

${ }^{19} \mathrm{~L}$. Ting and C. Tung, "Motion and decay of a vortex in a nonuniform stream," Phys. Fluids 8, 1039 (1965).

${ }^{20}$ L. Ting and R. Klein, Viscous Vortical Flows (Springer-Verlag, Berlin, 1991).

${ }^{21} \mathrm{M}$. D. Gunzburger, "Long time behavior of a decaying vortex," Z. Angew. Math. Mech. 53, 751 (1973).

${ }^{22}$ T. S. Lundgren, "Strained spiral vortex model for turbulent fine structure," Phys. Fluids 25, 2193 (1982).

${ }^{23} \mathrm{P}$. B. Rhines and W. R. Young, "How rapidly is a passive scalar mixed within closed streamlines?," J. Fluid Mech. 133, 133 (1983).

${ }^{24} \mathrm{~J}$. F. Lingevitch and A. J. Bernoff, "Advection of a passive scalar by a vortex couple in the small diffusion limit," J. Fluid Mech. 270, 219 (1994).

${ }^{25}$ A. Fannjiang and G. Papanicolaou, "Convection enhanced diffusion for periodic flows," SLAM J. Appl. Math. 54, 333 (1994).

${ }^{26} \mathrm{E}$. Knobloch and W. J. Merryfield, "Enhancement of diffusive transport in oscillatory flows," Astrophys. J. 401, 196 (1992).

${ }^{27}$ W. R. Young and S. Jones, "Shear dispersion," Phys. Fluids A 3, 1087 (1991). 